Bull. Chem. Soc. Ethiop. 2017, 31(2), 323-329.

ISSN 1011-3924

(c) 2017 Chemical Society of Ethiopia and The Authors

Printed in Ethiopia

DOI: http://dx.doi.org/10.4314/bcse.v31i2.13

\title{
GRINDING TECHNIQUE FOR THE TANDEM SYNTHESIS OF BIS COUMARINYL METHANES USING [BDBDMIm]Br-CAN
}

\author{
Mohammad Nikpassand $^{{ }^{*}}$, Leila Zare Fekri ${ }^{2}$ and Sanaz Sahrapeima ${ }^{1}$ \\ ${ }^{1}$ Department of Chemistry, Rasht Branch, Islamic Azad University, Rasht, Iran \\ ${ }^{2}$ Department of Chemistry, Payame Noor University, PO Box 19395-3697 Tehran, Iran
}

(Received March 4, 2016; revised September 25, 2017)

\begin{abstract}
Butane-1,4-diyl)bis(1,2-dimethyl-1 $H$-imidazole-3-ium)bromide ([BDBDMIm]Br) is easily used as an efficient and recyclable ionic liquid for the synthesis of bis coumarinyl methanes in the presence of a catalytic amount of ceric ammonium nitrate $(\mathrm{CAN})$ under grinding. All reactions are performed in the absence of solvent in excellent yield during short reaction time. Furthermore, the ionic liquid can be reused and recovered for several times without loss of activity. This method provides several advantages such as simple work-up, environmental friendliness and shorter reaction time along with high yields. All of synthesized compounds were characterized by infrared spectroscopy, ${ }^{1} \mathrm{H}$ and ${ }^{13} \mathrm{C}$ nuclear magnetic resonance spectroscopy and elemental analyses.
\end{abstract}

KEY WORDS: Grinding, Ionic liquid, CAN, 4-Hydroxycoumarin, Oxidation, One-pot

\section{INTRODUCTION}

Coumarin constitutes one of the major classes of naturally occurring compounds. In the wellknown family of coumarin derivatives, dimeric coumarins (also called biscoumarins) occupy an interesting position. Although some types of these compounds could be isolated from plants [1], we are interested in its chemistry because of its benefits as biologically active agents. It also represents the core structure of several molecules of pharmaceutical importance. Coumarin has been reported to serve as anti-cancer [2], anti-microbial [3], anti-inflammatory [4] and anticoagulant [5]. These pharmacological properties of coumarin encouraged us to synthesize some coumarin derivatives.

The use of ionic liquids as reaction media and catalyst can offer a solution to solvent emission and catalyst recycle problems. Ionic liquid possess the advantages like negligible vapor pressure, nonflamability, no miscibility with non-polar solvents, reasonable thermal and chemical stability and recyclability [6-8]. They dissolve many organic and inorganic substrates and are tunable to specific chemical tasks [9]. Recently, ionic liquids have been successfully employed as solvents with catalytic activity for a variety of reactions [10-12].

Looking for the eco-friendly alternatives to synthesize of bis coumarinylmethanes at mild and practical protocols, we report herein the synthesis of bis coumarinylmethanes using [BDBDMIm]Br-CAN under grinding as a green and novel method (Scheme 1).

\section{RESULTS AND DISCUSSION}

As part of our interest for the development of efficient and environmentally friendly procedures for the synthesis of heterocyclic and pharmaceutical compounds [13-16], an efficient, facile and solvent free procedure was introduced for the synthesis of bis coumarinylmethane through the reaction of one equivalent of synthesized aldehyde and two equivalent of 4-hydroxycoumarin in the presence of ionic liquid [BDBDMIm]Br. Although, synthesis of biscoumarins using ionic liquids such as sulfonic acid-functionalized pyridinium chloride [17], $\mathrm{RuCl}_{3} \cdot \mathrm{nH}_{2} \mathrm{O}$ [18],

*Corresponding author. E-mail: Nikpassand@iaurasht.ac.ir

This work is licensed under the Creative Commons Attribution 4.0 International License 
[bmim] $\mathrm{BF}_{4}$ [19], $\mathrm{SO}_{3} \mathrm{H}$-functionalized benzimidazolium cation [20], $\mathrm{NaHSO}_{4} / \mathrm{SiO}_{2} /$ Indion 190 resin [21], [MIM $\left.\left(\mathrm{CH}_{2}\right)_{4} \mathrm{SO}_{3} \mathrm{H}\right]\left[\mathrm{HSO}_{4}\right][22]$ and choline chloride-oxalic acid [23] was reported, however, in all of these reported methods aldehydes were used as substrate and most of these suffer from environmental pollution, expensive reagents or catalysts, long reaction time, exotic reaction conditions, unsatisfactory yields and complicated operations. In order to make this reaction simple and green, herein, we used ionic liquid [BDBDMIm] Br to the synthesis of bis coumarinylmethanes through the one-pot reaction of 4-hydroxycoumarin and various benzylalcohols at room temperature (Scheme 1).

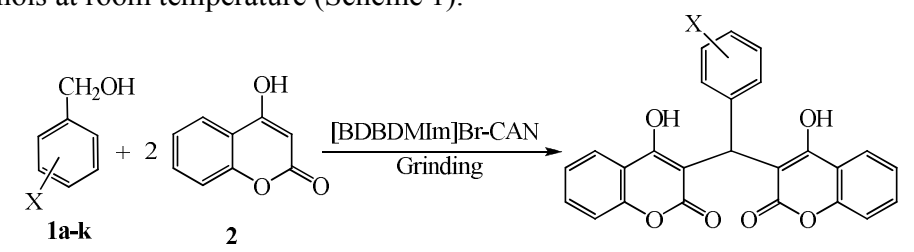<smiles></smiles>

3a-k

Scheme 1. Synthesis of bis-coumarinylmethanes using [BDBDMIm]Br-CAN under grinding.

To investigate the efficiency of catalyst, some of other catalyst was checked in the model reaction for the synthesis of 3a. All the reactions were carried out with catalytic amounts of catalysts. As shown in Table 1 the best results gained with $0.4 \mathrm{mmol}(4 \mathrm{mmol} \%)$ of [BDBDMIm]Br (Table 1; Entry 11).

Table 1. Effect of catalyst and catalyst amount for the synthesis of $3 \mathbf{a}$ using $0.55 \mathrm{~g}$ of CAN ${ }^{\mathrm{a}}$.

\begin{tabular}{|c|c|c|c|c|c|}
\hline Entry & Catalyst & $\begin{array}{c}\text { Catalyst amount/1 mmol of } \\
\text { aldehyde }\end{array}$ & $\begin{array}{c}\text { Reaction } \\
\text { condition }\end{array}$ & Time (min) & Yield (\%) \\
\hline 1 & $\mathrm{H}_{2} \mathrm{SO}_{4}$ & 4 drops & Reflux & 240 & 55 \\
\hline 2 & $\mathrm{I}_{2}$ & $0.2 \mathrm{mmol}$ & Reflux & 360 & 63 \\
\hline 3 & Montmorillonite $\mathrm{K} 10$ & $0.2 \mathrm{~g}$ & Reflux & 240 & 72 \\
\hline 4 & Montmorillonite KSF & $0.2 \mathrm{~g}$ & Reflux & 240 & 72 \\
\hline 5 & $\mathrm{Fe}_{3} \mathrm{O}_{4}$ & $0.2 \mathrm{mmol}$ & Reflux & 180 & 78 \\
\hline 6 & $\mathrm{ZnCl}_{2}$ & $0.2 \mathrm{mmol}$ & Reflux & 210 & 70 \\
\hline 7 & {$[\mathrm{BMIM}] \mathrm{Br}$} & $0.4 \mathrm{mmol}$ & Neat, r.t. & 60 & 80 \\
\hline 8 & {$[\mathrm{BMIM}] \mathrm{OH}$} & $0.4 \mathrm{mmol}$ & Neat, r.t. & 60 & 76 \\
\hline 9 & {$[\mathrm{BDBDMIm}] \mathrm{Br}$} & $0.2 \mathrm{mmol}$ & Neat, r.t. & 30 & 85 \\
\hline 10 & {$[\mathrm{BDBDMIm}] \mathrm{Br}$} & $0.4 \mathrm{mmol}$ & Neat, r.t. & 20 & 94 \\
\hline 11 & {$[\mathrm{BDBDMIm}] \mathrm{Br}$} & $0.6 \mathrm{mmol}$ & Neat, r.t. & 20 & 98 \\
\hline
\end{tabular}

${ }^{\mathrm{a}}$ Solvent in the entries 1-6 was water.

The reaction yields in the presence of ionic liquid were increased and the reaction times were dramatically shortened to $15-30 \mathrm{~min}$. Therefore, using of ionic liquid exhibited some advantages over the classical condition by improving the reaction yields and reducing the reaction time (Scheme 2).

In the first step, we select 1a as a model substrate and treated it with 4-hydroxycoumarin in the presence of $0.4 \mathrm{mmol}$ [BDBDMIm] $\mathrm{Br}$ and $0.55 \mathrm{~g}$ of CAN under solvent free reaction. The same reaction was carried out with $0.2,0.4$ and $0.6 \mathrm{mmol}$ of the ionic liquid, respectively. The best results were obtained using $0.6 \mathrm{mmol}$ of the catalyst with complete conversion within 20 min and in $98 \%$ yields. 


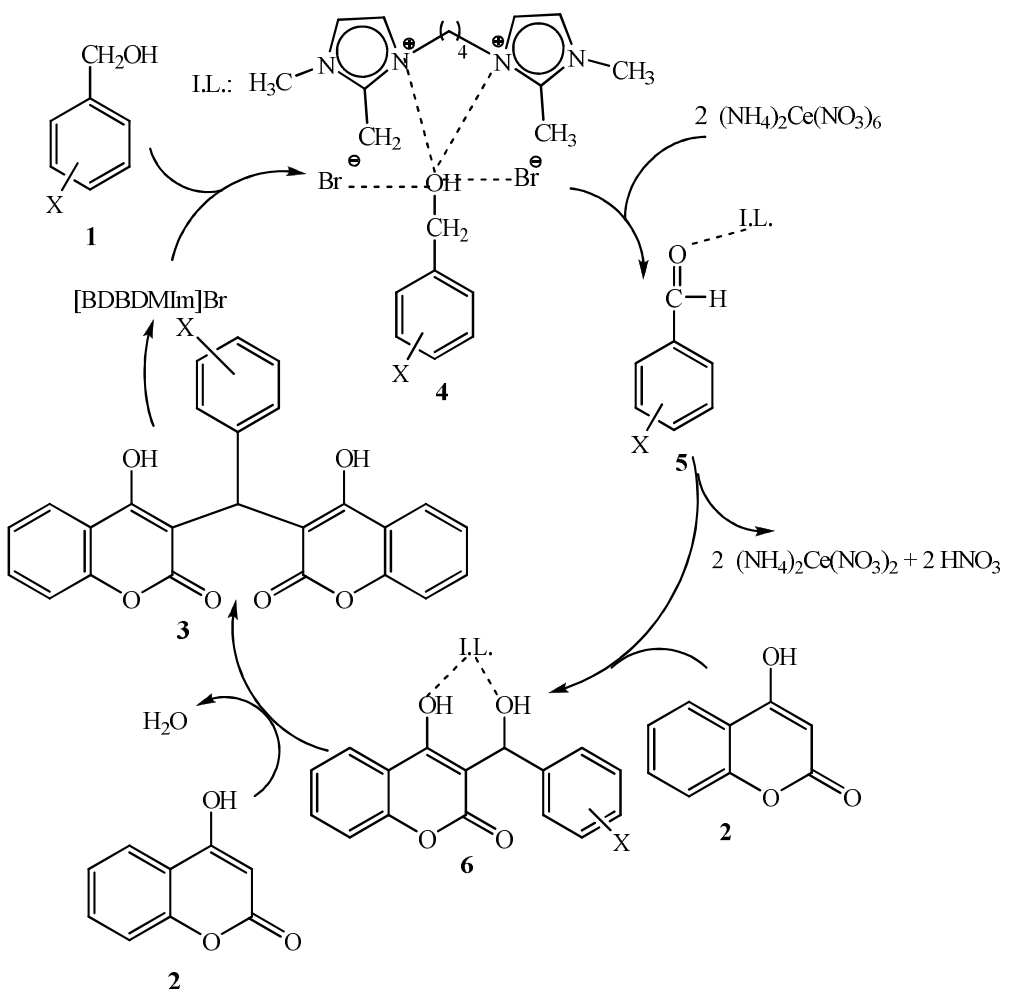

Scheme 2. A possible mechanism for the synthesis of bis coumarinylmethanes.

To investigation of generality of this method some benzyl alcohols bearing electron withdrawing or electron releasing substituents were checked. The results were presented in Table 2 .

Table 2. Synthesis of biscoumarinylmethane 3a-k using [BDBDMIm] $\mathrm{Br}$ at room temperature. ${ }^{a}$

\begin{tabular}{|c|c|c|c|c|c|c|}
\hline \multirow[t]{2}{*}{ Compound } & \multirow[t]{2}{*}{$\mathrm{X}$} & \multirow[t]{2}{*}{ Time (min) } & \multirow[t]{2}{*}{ Yield $(\%)^{b}$} & \multicolumn{2}{|c|}{$\mathrm{Mp}\left({ }^{\circ} \mathrm{C}\right)$} & \multirow[t]{2}{*}{ Ref } \\
\hline & & & & Found & Reported & \\
\hline $3 \mathbf{a}$ & $4-\mathrm{NO}_{2}$ & 20 & 98 & $231-233$ & $232-234$ & [24] \\
\hline $\mathbf{3 b}$ & $3-\mathrm{NO}_{2}$ & 20 & 98 & $233-235$ & $234-236$ & [25] \\
\hline $3 c$ & $2-\mathrm{NO}_{2}$ & 45 & 89 & $201-203$ & 202 & [24] \\
\hline 3d & $4-\mathrm{Br}$ & 25 & 92 & $265-267$ & 267 & [25] \\
\hline $3 e$ & $3-\mathrm{Br}$ & 60 & 90 & $286-288$ & 287 & [24] \\
\hline 3f & $4-\mathrm{Cl}$ & 30 & 92 & $255-256$ & $256-258$ & [24] \\
\hline $3 g$ & $3-\mathrm{Cl}$ & 45 & 90 & $254-256$ & - & - \\
\hline $3 \mathrm{~h}$ & $2-\mathrm{Cl}$ & 60 & 87 & $223-224$ & 224 & {$[21]$} \\
\hline $3 \mathbf{i}$ & $4-\mathrm{F}$ & 20 & 92 & $212-214$ & 214 & [25] \\
\hline $3 \mathbf{j}$ & 4-OH & 45 & 90 & $223-225$ & $22-224$ & [21] \\
\hline $3 \mathbf{k}$ & $3-\mathrm{OH}$ & 45 & 90 & $218-220$ & - & - \\
\hline 31 & $2-\mathrm{OH}$ & 60 & 82 & $253-255$ & $254-256$ & {$[24]$} \\
\hline $3 \mathrm{~m}$ & $\mathrm{H}$ & 60 & 90 & $254-255$ & $256-258$ & {$[24]$} \\
\hline
\end{tabular}

${ }^{a}$ All products were characterized by their physical constant, comparison with authentic samples, IR and NMR spectroscopy. ${ }^{b}$ Yields based upon starting aldehyde.

Bull. Chem. Soc. Ethiop. 2017, 31(2) 
In order to investigate the reusability of [BDBDMIm]Br, a recycling experiment was conducted. The catalyst could be recycled up to five times without significant loss of activity (Table 3).

Table 3. Evaluation of reusability of [BDBDMIm]Br for the synthesis of 3a.

\begin{tabular}{|c|c|c|c|c|c|c|}
\hline Run & 1 & 2 & 3 & 4 & 5 & 6 \\
\hline Time (min) & 20 & 20 & 20 & 21 & 20 & 25 \\
\hline Yield (\%) & 98 & 98 & 96 & 98 & 97 & 86 \\
\hline
\end{tabular}

A possible mechanism for the synthesis of bis coumarinylmethane derivatives (Scheme 2) is proposed. It is assumed that the reaction may proceed initially through full activation by polarization of benzyl alcohols with [BDBDMIm] $\mathrm{Br}$ to form intermediate 4. Next, benzyl alcohols convert to benzaldehydes 5 by oxidation with $\mathrm{CAN}\left(\left(\mathrm{NH}_{4}\right)_{2} \mathrm{Ce}\left(\mathrm{NO}_{3}\right)_{6}\right)$. In fact oxidation state of $\mathrm{Ce}(\mathrm{IV})$ changes to oxidation state in $\left(\mathrm{NH}_{4}\right)_{2} \mathrm{Ce}\left(\mathrm{NO}_{3}\right)_{5}(\mathrm{Ce}(\mathrm{III}))$ and nitric acid $[26,27]$. Then nucleophilic addition of 4-hydroxycoumarin to intermediate $\mathbf{5}$ affords $\mathbf{6}$. Finally, nucleophilic attack of second 4-hydroxycoumarin to compound $\mathbf{6}$ and dehydration lead to compound $\mathbf{3}$.

\section{CONCLUSIONS}

In conclusion, we have investigated the ionic liquid [BDBIM] Br as a mild and efficient catalyst for the synthesis of substituted biscoumarin compounds. The remarkable advantages offered by this method are: catalyst is inexpensive, non-toxic, easy handling and reusable, simple work-up procedure, short reaction time, high yields of product with better purity and green aspect by avoiding toxic catalyst and hazardous solvent. To the best of our knowledge this is the first report on synthesis of biscoumarin compounds using ionic liquid [BDBDMIm]Br.

\section{EXPERIMENTAL}

Melting points were measured on an Electrothermal 9100 apparatus. Chemicals were purchased from Merck and Fluka and used as purchased. Melting points were measured on an Electrothermal 9100 apparatus and are uncorrected. ${ }^{1} \mathrm{H}$ NMR and ${ }^{13} \mathrm{C}$ NMR spectra were obtained on a Bruker DRX 500 Avance spectrometer in $\mathrm{CDCl}_{3}$ and DMSO- $\mathrm{d}_{6}$ as solvent and with TMS as internal standard. FT-IR spectra were recorded on a Shimadzu FT-IR-8400S spectrometer. Elemental analyses were recorded on a Carlo-Erba EA1110CNNO-S analyzer.

\section{General procedure for preparation of compounds $\mathbf{3 a - k}$}

A mixture containing benzyl alcohol $(1 \mathrm{mmol})$, 4-hydroxycoumarin $(2 \mathrm{mmol})$ and $4 \mathrm{mmol} \%$ of [BDBDMIm]Br [16] and $0.55 \mathrm{~g}$ of CAN was stirred at room temperature for the required reaction times. The progress of the reaction was monitored by TLC (EtOAc : petroleum ether 1:2). Having completed the reaction, we extracted the product with $\mathrm{CHCl}_{3} / \mathrm{H}_{2} \mathrm{O}$. After separation of phases and evaporation of the organic phase and recrystallization of the residue, the pure product was obtained. The aqueous phase was concentrated under reduced pressure, washed with $\mathrm{Et}_{2} \mathrm{O}$, and evaporated under reduced pressure to recover the ionic liquid for subsequent use.

\section{Selected spectral data}

4-Hydroxy-3-((4-hydroxy-2-oxo-2H-chromen-3-yl)(4-nitrophenyl)methyl)-2H-chromen-2-one (3a). Yellow solid, IR (KBr) $\left(v_{\max }, \mathrm{cm}^{-1}\right): 3433,1662,1561,1608,1520,1348,1103 ;{ }^{1} \mathrm{H}$ NMR 
(400 MHz, DMSO- $\left.d_{6}\right) \delta: 6.40(\mathrm{~s}, 1 \mathrm{H}), 6.84$ (s, br., 2H), 7.30-7.42 (m, 6H), 7.58 (s, br., 2H), $7.88(\mathrm{~d}, J=6.7 \mathrm{~Hz}, 2 \mathrm{H}), 8.08(\mathrm{~d}, J=6.7 \mathrm{~Hz}, 2 \mathrm{H}) ;{ }^{13} \mathrm{C}$ NMR $\left(100 \mathrm{MHz}\right.$, DMSO- $\left.d_{6}, \delta / \mathrm{ppm}\right)$ : $36.8,103.4,116.0,118.5,123.3,123.7,124.1,128.2$, 131.9, 145.6, 150.1, 152.5, 164.5, 166.4. Anal calc. for $\mathrm{C}_{25} \mathrm{H}_{15} \mathrm{NO}_{8}$ : C, 65.65; H, 3.31; N, 3.06. Found: C, 65.63; H, 3.29; N, 3.08.

4-Hydroxy-3-((4-hydroxy-2-oxo-2H-chromen-3-yl)(3-nitrophenyl)methyl)-2H-chromen-2-one (3b). Cream solid, IR (KBr) $\left(v_{\max }, \mathrm{cm}^{-1}\right): 3428,3079,1658,1611,1564,1528,1347,1102,1058$. ${ }^{1} \mathrm{H}$ NMR $\left(400 \mathrm{MHz}, \mathrm{DMSO}-d_{6}\right) \delta: 6.39(\mathrm{~s}, 1 \mathrm{H}), 7.27-7.35(\mathrm{~m}, 4 \mathrm{H}), 7.50-7.57(\mathrm{~m}, 4 \mathrm{H}), 7.86(\mathrm{~d}$, $1 \mathrm{H}, J=8.0 \mathrm{~Hz}), 7.92(\mathrm{~s}, 1 \mathrm{H}), 8.03(\mathrm{~d}, 1 \mathrm{H}, J=8.0 \mathrm{~Hz})$.

4-Hydroxy-3-((4-hydroxy-2-oxo-2H-chromen-3-yl)(2-nitrophenyl)methyl)-2H-chromen-2-one (3c). Cream solid, IR (KBr) $\left(v_{\max }, \mathrm{cm}^{-1}\right): 3429,3075,2930,1656,1609,1562,1354,1522,1069$; ${ }^{1} \mathrm{H}$ NMR (400 MHz, DMSO- $\left.d_{6}\right) \delta: 5.14(\mathrm{~s}, 1 \mathrm{H}), 7.26-7.33(\mathrm{~m}, 4 \mathrm{H}), 7.40(\mathrm{~d}, 2 \mathrm{H}, J=7.6 \mathrm{~Hz})$, $7.42-7.58(\mathrm{~m}, 3 \mathrm{H}), 7.66(\mathrm{~d}, 1 \mathrm{H}, J=7.6 \mathrm{~Hz}), 7.84(\mathrm{~d}, 2 \mathrm{H}, J=8.0 \mathrm{~Hz}) ;{ }^{13} \mathrm{C}$ NMR $(100 \mathrm{MHz}$, DMSO- $\left.d_{6}\right)$ : $34.4,103.6,116.1,117.7,123.7,123.8,124.1,127.2,130.0,131.9,132.2,134.7$, 149.5, 152.3, 163.4, 165.0.

3-((4-Fluorophenyl)(4-hydroxy-2-oxo-2H-chromen-3-yl)methyl)-4-hydroxy-2H-chromen-2-one (3i). Cream solid, IR (KBr) $\left(v_{\max }, \mathrm{cm}^{-1}\right): 3424,1670,1610,1561,1099 ;{ }^{1} \mathrm{H}$ NMR $(400 \mathrm{MHz}$, DMSO- $\left.d_{6}\right) \delta: 6.36(\mathrm{~s}, 1 \mathrm{H}), 7.05(\mathrm{t}, 2 \mathrm{H}, J=8.0 \mathrm{~Hz}), 7.19-7.29(\mathrm{~m}, 2 \mathrm{H}), 7.31(\mathrm{t}, 2 \mathrm{H}, J=8.8 \mathrm{~Hz})$, $7.37(\mathrm{~d}, 2 \mathrm{H}, J=8.0 \mathrm{~Hz}), 7.59(\mathrm{td}, 2 \mathrm{H}, J=8.4,2.0 \mathrm{~Hz}), 7.91(\mathrm{dd}, 2 \mathrm{H}, J=8.0,2.0 \mathrm{~Hz}, 2 \mathrm{H}) ;{ }^{13} \mathrm{C}$ NMR (100 MHz, DMSO- $d_{6}$ ): 35.5, 104.3, 115.0, 116.1, 117.7, 124.0, 128.8, 132.2, 132.8, $135.7,152.3,159.5,161.9,165.1$.

3-((4-Bromophenyl)(4-hydroxy-2-oxo-2H-chromen-3-yl)methyl)-4-hydroxy-2H-chromen-2-one (3d). Cream solid, IR (KBr) $\left(v_{\max }, \mathrm{cm}^{-1}\right): 3421,3071,2938,1669,1610,1561,1488,1096,765$. ${ }^{1} \mathrm{H}$ NMR $\left(400 \mathrm{MHz}, \mathrm{DMSO}-d_{6}\right) \delta: 6.33(\mathrm{~s}, 1 \mathrm{H}), 7.12(\mathrm{~d}, 2 \mathrm{H}, J=8.0 \mathrm{~Hz}), 7.34-7.42(\mathrm{~m}, 4 \mathrm{H})$, 7.59 (t, $4 \mathrm{H}, J=7.6 \mathrm{~Hz}), 7.90(\mathrm{~d}, 2 \mathrm{H}, J=7.6 \mathrm{~Hz}) .{ }^{13} \mathrm{C}$ NMR (100 MHz, DMSO-d6): 35.8, 104.0, $116.1,117.9,118.7,123.9,124.0,129.2,131.0,132.1,139.7,152.3,164.8,165.4$.

3-((3-Bromophenyl)(4-hydroxy-2-oxo-2H-chromen-3-yl)methyl)-4-hydroxy-2H-chromen-2-one (3e). Pink solid, IR (KBr) $\left(v_{\max }, \mathrm{cm}^{-1}\right): 3421,3074,1664,1609,1561,1498,1095,765 .{ }^{1} \mathrm{H}$ NMR $\left(400 \mathrm{MHz}, \mathrm{DMSO}-d_{6}\right) \delta: 6.35(\mathrm{~s}, 1 \mathrm{H}), 7.10(\mathrm{~s}, \mathrm{br}, 3 \mathrm{H}), 7.31-7.36(\mathrm{~m}, 7 \mathrm{H}), 7.58(\mathrm{t}, 2 \mathrm{H}, J=7.2$ $\mathrm{Hz}), 7.90(\mathrm{~d}, 2 \mathrm{H}, J=7.2 \mathrm{~Hz}) .{ }^{13} \mathrm{C}$ NMR (100 MHz, DMSO-d6): 36.1, 103.7, 116.0, 118.1, 121.7, $123.8,124.0,126.1,128.6,129.4,130.3,132.0,143.6,152.3,164.7,165.7)$.

3-((4-Chlorophenyl) (4-hydroxy-2-oxo-2H-chromen-3-yl)methyl)-4-hydroxy-2H-chromen-2-one (3f). Cream solid, $300{ }^{\circ} \mathrm{C}$, IR $(\mathrm{KBr})\left(\mathrm{v}_{\max }, \mathrm{cm}^{-1}\right): 3434,1668,1613,1562,1495,1093,767 .{ }^{1} \mathrm{H}$ NMR (400 MHz, DMSO- $\left.d_{6}\right) \delta: 6.28(\mathrm{~s}, 1 \mathrm{H}), 7.14(\mathrm{~d}, 2 \mathrm{H}, J=8.0 \mathrm{~Hz}), 7.24-7.35(\mathrm{~m}, 6 \mathrm{H}), 7.57$ (t, $2 \mathrm{H}, J=8.0 \mathrm{~Hz}), 7.88(\mathrm{~d}, 2 \mathrm{H}, J=8.0 \mathrm{~Hz})$.

3-((3-Chlorophenyl)(4-hydroxy-2-oxo-2H-chromen-3-yl)methyl)-4-hydroxy-2H-chromen-2-one (3g). Pink solid, IR (KBr) $\left(v_{\max }, \mathrm{cm}^{-1}\right): 3398,3074,1665,1610,1562,1489,1098,764 .{ }^{1} \mathrm{H}$ NMR $\left(400 \mathrm{MHz}, \mathrm{DMSO}-d_{6}\right) \delta: 6.34(\mathrm{~s}, 1 \mathrm{H}), 7.12-7.37(\mathrm{~m}, 1 \mathrm{H}), 7.59$ (t, 2H, J=7.2 Hz), 7.92 (d, 2H, $J=8.0 \mathrm{~Hz})$.

3-((2-Chlorophenyl)(4-hydroxy-2-oxo-2H-chromen-3-yl)methyl)-4-hydroxy-2H-chromen-2-one (3h). Yellow solid, IR (KBr) $\left(v_{\max }, \mathrm{cm}^{-1}\right): 3429,1658,1612,1562,1495,1053,759 .{ }^{1} \mathrm{H}$ NMR $\left(400 \mathrm{MHz}, \mathrm{DMSO}-d_{6}\right) \delta: 6.16(\mathrm{~s}, 1 \mathrm{H}), 7.16-7.23(\mathrm{~m}, 2 \mathrm{H}), 7.27-7.37(\mathrm{~m}, 6 \mathrm{H}), 7.56(\mathrm{t}, 1 \mathrm{H}, J=7.6$ $\mathrm{Hz}), 7.89(\mathrm{~d}, 1 \mathrm{H}, J=8.0 \mathrm{~Hz})$. 
4-Hydroxy-3-((4-hydroxy-2-oxo-2H-chromen-3-yl)(3-hydroxyphenyl)methyl)-2H-chromen-2one $(\mathbf{3 k})$. Brown solid, IR (KBr) $\left(v_{\max }, \mathrm{cm}^{-1}\right): 3397,1655,1612,1569,1489,1054 .{ }^{1} \mathrm{H}$ NMR $\left(400 \mathrm{MHz}, \mathrm{DMSO}-d_{6}\right) \delta: 6.28(\mathrm{~s}, 1 \mathrm{H}), 6.52-6.58(\mathrm{~m}, 3 \mathrm{H}), 7.0(\mathrm{t}, 1 \mathrm{H}, J=8.0 \mathrm{~Hz}), 7.30-7.37(\mathrm{~m}$, $4 \mathrm{H}), 7.59(\mathrm{t}, 2 \mathrm{H}, J=8.0 \mathrm{~Hz}), 7.91(\mathrm{~d}, 2 \mathrm{H}, J=8.0 \mathrm{~Hz})$.

4-Hydroxy-3-((4-hydroxy-2-oxo-2H-chromen-3-yl)(2-hydroxyphenyl)methyl)-2H-chromen-2one (3l). Cream solid, IR (KBr) $\left(v_{\max }, \mathrm{cm}^{-1}\right): 3251,3078,1710,1671,1634,1571,1220 .{ }^{1} \mathrm{H}$ NMR (400 MHz, DMSO-d $\left.)_{6}\right) \delta: 5.74(\mathrm{~s}, 1 \mathrm{H}), 7.14(\mathrm{t}, 1 \mathrm{H}, J=7.8 \mathrm{~Hz}), 7.19(\mathrm{~d}, 1 \mathrm{H}, J=8.0 \mathrm{~Hz})$, $7.31-7.45(\mathrm{~m}, 5 \mathrm{H}), 7.43(\mathrm{~d}, 1 \mathrm{H}, J=8.4 \mathrm{~Hz}), 7.48(\mathrm{~d}, 1 \mathrm{H}, J=8.0 \mathrm{~Hz}), 7.59(\mathrm{t}, 1 \mathrm{H}, J=8.0 \mathrm{~Hz})$, 7.69 (t, 1H, $J=7.4 \mathrm{~Hz}$ ), 8.07 (brs, 1H), 8.08 (d, 1H, $J=7.6) .{ }^{13} \mathrm{C}$ NMR (100 MHz, DMSO-d6): 28.7, 113.8, 116.1, 116.3, 116.5, 122.7, 124.0, 124.6, 125.4, 128.7, 132.3, 132.6, 149.2, 152.0, $156.3,160.5$.

\section{ACKNOWLEDGEMENT}

Financial support from the Research Council of Islamic Azad University of Rasht branch is sincerely acknowledged.

\section{REFERENCES}

1. Borges, F.; Roleira, F.; Milhazes, N.; Santana, L.; Uriarte, E. Simple coumarins and analogues in medicinal chemistry: occurrence, synthesis and biological activity. Curr. Med. Chem. 2005, 12, 887-916.

2. Lacy, A.; O'Kennedy, R. studies on coumarins and coumarin-related compounds to determine their therapeutic role in the treatment of cancer. Curr. Pharm. Design 2004, 10, 3797-3811.

3. Chougala, B.M.; Shastri, S.L.; Holiyachi, M.; Shastri, L.A.; More, S.S.; Ramesh, K.V. Synthesis, anti-microbial and anti-cancer evaluation study of 3-(3-benzofuranyl)-coumarin derivatives. Med. Chem. Res. 2015, 24, 4128-4138.

4. Su, C.X.; Mouscadet, J.F.; Chiang, C.C.; Tsai, H.J.; Hsu, L.Y. HIV-1 integrase inhibition of biscoumarin analogues. Chem. Pharm. Bull. 2006, 54, 682-686.

5. Choudhary, M.I.; Fatima, N.; Khan, K.M.; Jalil, S.; Iqbal, S.; Atta-ur-Rahman. New biscoumarin derivatives-cytotoxicity and enzyme inhibitory activities. Bioorg. Med. Chem. 2006, 14, 8066-8072.

6. Roy, S.R.; Jadhavar, P.S.; Seth, K.; Sharma, K.K.; Chakraborti, A.K. Organocatalytic application of ionic liquids: $[\mathrm{bmim}]\left[\mathrm{MeSO}_{4}\right]$ as a recyclable organocatalyst in the multicomponent reaction for the preparation of dihydropyrimidinones and -thiones. Synthesis 2011, 14, 2261-2267.

7. Roy, S.R.; Chakraborti, A.K. Supramolecular assemblies in ionic liquid catalysis for AzaMichael reaction. Org. Lett. 2010, 12, 3866-3869.

8. Chakraborti, A.K.; Roy, S.R. On catalysis by ionic liquids. J. Am. Chem. Soc. 2009, 131, 6902-6903.

9. Lee, J.K.; Kim, M.J. Ionic liquid-coated enzyme for biocatalysis in organic solvent. J. Org. Chem. 2002, 67, 6845-6847.

10. Kefayati, H.; Asghari, F.; Khanjanian, R. 1-Methylimidazolium hydrogen sulfate/chlorotrimethylsilane: An effective catalytic system for the synthesis of 3,4dihydropyrimidin-2(1H)-ones and hydroquinazoline-2,5-diones. J. Mol. Liq. 2012, 172, 147 151.

11. Yao, L.; Liu, Sh.; Li, L.; Yu, Sh.; Liu, F.; Song, Z. Synthesis of hydroxymethylfurfural from sucrose using Brönsted-Lewis acidic ionic liquid. Bull. Chem. Soc. Ethiop. 2016, 30, $283-$ 288. 
12. Hu, Y.L.; Xie, Y.B.; Li, D.J. Efficient Baeyer-Villiger electro-oxidation of ketones with molecular oxygen using an activated carbon fiber electrode in ionic liquid [bmim][OTf]. Bull. Chem. Soc. Ethiop. 2016, 30, 297-306.

13. Nikpassand, M.; Zare, L.; Saberi, M. Ultrasound-assisted $L$-proline catalyzed synthesis of novel derivatives of azo-linked dihydropyridines. Monatsch. Chem. 2012, 143, 289-293.

14. Zare Fekri, L.; Nikpassand, M.; Hassanpour, K. Green aqueous synthesis of mono, bis and trisdihydropyridines using nano $\mathrm{Fe}_{3} \mathrm{O}_{4}$ under ultrasound irradiation. Curr. Org. Syn. 2015, 12, 76-79.

15. Zare, L.; Mahmoodi, N.O.; Yahyazadeh, A.; Nikpassand, M. Ultrasound-promoted regio and chemoselective synthesis of pyridazinones and phthalazinones catalyzed by ionic liquid [bmim] Br/AlCl . Ultrason Sonochem. 2012, 19, 740-744.

16. Nikpassand, M.; Zare Fekri, L.; Farokhian, P. Synthesis of novel derivatives of benzoxazole in bis-ionic liquid [BDBDIm]Br. Synth. Commun. 2016, 45, 2303-2310.

17. Zolfigol, M.A.; Mousavi-Zare, A.R.; Zarei, M. Friedel-Crafts alkylation of 4hydroxycoumarin catalyzed by sulfonic-acid-functionalized pyridinium chloride as a new ionic liquid. Comp. Rend. Chim. 2014, 17, 1264-1267.

18. Tabatabaeian, K.; Heidari, H.; Khorshidi, A.; Mamaghani, M.; Mahmoodi, N.O. Synthesis of biscoumarin derivatives by the reaction of aldehydes and 4-hydroxycoumarin using ruthenium (III) chloride hydrate as a versatile homogeneous catalyst. J. Serb. Chem. Soc. 2012, 77, 407-413.

19. Khurana, J.M.; Kumar, S. Ionic liquid: an efficient and recyclable medium for the synthesis of octahydroquinazolinone and biscoumarin derivatives. Monatsh. Chem. 2010, 141, 561564.

20. Li, W.; Wang, Y.; Wang, Z.; Dai, L.; Wang, Y. Novel $\mathrm{SO}_{3} \mathrm{H}$-functionalized ionic liquids based on benzimidazolium cation: Efficient and recyclable catalysts for one-pot synthesis of biscoumarin derivatives. Catal. Lett. 2010, 141, 1651-1658.

21. Padalkar, V.; Phatangare, K.; Takale, S.; Pisal, R.; Chaskar, A. Silica supported sodium hydrogen sulfate and Indion 190 resin: An efficient and heterogeneous catalysts for facile synthesis of bis-(4-hydroxycoumarin-3-yl) methanes. J. Saudi Chem. Soc. 2015, 19, 42-45.

22. Tavakoli-Hoseini, N.; Heravi, M.M.; Bamoharram, F.F.; Davoodnia, A.; Ghassemzadeh, M. An unexpected tetracyclic product isolated during the synthesis of biscoumarins catalyzed by $\left[\mathrm{MIM}\left(\mathrm{CH}_{2}\right)_{4} \mathrm{SO}_{3} \mathrm{H}\right]\left[\mathrm{HSO}_{4}\right]$ : Characterization and X-ray crystal structure of 7-(2-hydroxy4-oxo-4H-chromen-3-yl)-6H,7H-chromeno[4,3-b]chromen-6-one. J. Mol. Liq. 2011, 163, 122-127.

23. Yadav, U.N.; Shankarling, G.S. Room temperature ionic liquid choline chloride-oxalic acid: A versatile catalyst for acid-catalyzed transformation in organic reactions. J. Mol. Liq. 2014, 191, 137-141.

24. Shamsaddini, A.; Sheikhhosseini, E. Synthesis of 3,3-arylidene bis (4-hydroxy coumarin) catalyzed by $p$-dodecylbenzenesulfonic acid (DBSA) in aqueous media and microwave irradiation. Int. J. Org. Chem. 2014, 4, 135-141.

25. Završnik, D.; Muratović, S.; Makuc, D.; Plavec, J.; Cetina, M.; Nagl, A.; Clercq, E.D.; Balzarini, J.; Mintas, M. Benzylidene-bis-(4-hydroxycoumarin) and benzopyrano-coumarin derivatives: Synthesis, ${ }^{1} \mathrm{H} /{ }^{13} \mathrm{C}$-NMR conformational and $\mathrm{X}$-ray crystal structure studies and in vitro antiviral activity evaluations. Molecules 2001, 16, 6023-6040.

26. Nair, V.; Deepthi, A. Cerium(IV) ammonium nitrate - a versatile single-electron oxidant. Chem. Rev. 2007, 107, 1862-1891.

27. Trahanovsky, W.S.; Young, L.B.; Brown, G.L. Cerium(III) bromate as a new reagent in oxidation of organic compounds. J. Org. Chem. 1967, 32, 3865-3868. 